\section{EDUCATION}

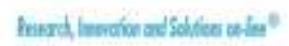

Electronic Journal of Research

in Educational Psychology

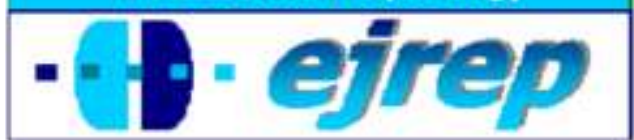

\title{
Las habilidades sociales en adolescentes tempranos de diferentes contextos socioeconómicos
}

\section{Coronel, Claudia Paola ${ }^{1}$; Levin, Mariel $^{2}$ y Mejail, Sergio ${ }^{3}$}

${ }^{1}$ Facultad de Psicología. Universidad Nacional de Tucumán, Argentina.

${ }^{2}$ Universidad del Norte Santo Tomás de Aquino. Tucumán, Argentina.

${ }^{3}$ Facultad de Psicología. Universidad Nacional de Tucumán, Argentina

\section{Argentina}




\section{Resumen}

Introducción. La adolescencia es un periodo del ciclo vital en el que las habilidades sociales son un factor fundamental para el ajuste social. Los comportamientos prosociales favorecen el desarrollo de la autoestima y el bienestar psicológico.

Método. El presente estudio tuvo como finalidad analizar y comparar las habilidades sociales en adolescentes de Tucumán, Argentina, provenientes de contextos de nivel socioeconómico alto y bajo; y determinar la prevalencia de las habilidades sociales facilitadoras o inhibidoras de la socialización. La muestra estuvo constituida por 283 adolescentes de 11 y 12 años, escolarizados. Se realizó un estudio descriptivo - explicativo con un muestreo intencional. Se empleó la Batería de Socialización BAS-3 y una encuesta Sociodemográfica.

Resultados. Se analizaron las habilidades sociales de los participantes según nivel socioeconómico -alto o bajo- y según sexo.

Discusión o Conclusión: Se hallaron diferencias significativas entre los grupos estudiados en cuanto a la prevalencia de las habilidades sociales facilitadoras de la socialización.

Palabras Clave: evaluación - habilidades sociales - adolescentes - nivel socioeconómico 


\title{
Social skills: an investigation with young adolescents from different socioeconomic contexts
}

\begin{abstract}
Introduction. Adolescence is a stage in the lyfe cycle where social abilities are a crucial factor in social adjustment. Prosocial behaviour contributes to the development of self-esteem and psychological well-being.

Method. The aim of this study was to analyze and compare social abilities in adolescents of low and high socioeconomic status, from Tucuman, Argentina and to determine whether the social skills that contribute to socialization or the ones that inhibit it prevail. The sample was constituted by 28311 and 12-year-old adolescents who attend school. A descriptiveexplanatory study with purposive sampling was carried out. The Socialization Battery BAS-3 and a sociodemographic survey were used.
\end{abstract}

Results: The participants' social abilities were analysed according to their socioeconomic status -low or high- and by sex.

Discussion and conclusión. Significant differences in the prevalence of social skills which contribute to socialization were found in the groups studied.

Keywords: evaluation - social abilities - adolescents - socioeconomic status

Received: 02/05/11 Initial Acceptance: 02/15/11 Definitive Acceptance: 03/13/11 


\section{Introducción}

La adolescencia es un fenómeno evolutivo socialmente situado, cultural e históricamente determinado (Ávila-Espada, Jiménez-Gómez, \& González 1996; Garagordobil, 2008; Musito, Buelga, Lila \& Cava, 2004). Griffa \& Moreno (2005) caracterizan al adolescente posmoderno como un sujeto inserto en una cultura del cambio. El desafío actual es cómo pensar la socialización de adolescentes en los inicios de esta etapa del ciclo vital. La familia y los pares, actúan como factores para el desarrollo social del adolescente. Aparecen nuevos modos de relación como las redes sociales vía Internet, a la que padres y docentes no suelen acceder y les resulta difícil comprender (Urresti, 2008). Sin embargo, es innegable la importancia del grupo de pares en el proceso de socialización. El ingreso a la escuela secundaria marca la aparición de nuevas reglas, nuevos modos y nuevos espacios de encuentro (Díaz-Aguado, 2005). No obstante, la familia influye en cómo los adolescentes atraviesan las nuevas tareas propias de su etapa.

Definir a las habilidades sociales (HHSS, en adelante) resulta complejo, debido a la naturaleza pluriconceptual de las mismas. En la literatura se encuentran diversas acepciones, tales como habilidades de interacción social (Garaigordobil, 2004, 2006; Monjas, 2000); habilidades sociales (Gil \& León, 1998; Trianes, 1996); asertividad (Wolpe, 1977). Además, se une a esta dificultad el carácter contextual de las mismas; esto es, lo que se considera aceptable a nivel social varia de un contexto a otro, de una cultura a otra. Tal como plantea Fernández Ballesteros (1994) existe una acentuada dificultad para acordar una definición de Habilidades Sociales (HHSS) por cuanto están referidas siempre a un contexto específico. Para Caballo $(1987,1993)$ las HHSS son un conjunto de conductas emitidas por un individuo en un contexto interpersonal que expresa sentimientos, actitudes, deseos, opiniones o derechos de ese individuo de un modo adecuado a la situación, respetando esas conductas en los demás; comportamientos con los que generalmente resuelve problemas inmediatos mientras minimiza la probabilidad de futuras dificultades. Para Monjas (2000) las habilidades son las "conductas o destrezas sociales específicas requeridas para ejecutar competentemente una tarea de índole interpersonal. Implica un conjunto de comportamientos adquiridos y aprendidos y no un rasgo de personalidad. Son un conjunto de comportamientos interpersonales complejos que se ponen en juego en la interacción con otras personas" (p. 28). Sin embargo, según Pelechano (1996), cabría diferenciar entre competencia social (éxito social, reconocimiento social de los méritos personales) y competencia interpersonal (reconocimiento individual de personas más 
que de instituciones). Se trata, en este último caso, del logro de una confianza personal, de ayuda hacia los demás y de un referente personal más que social. Según McFall (1982) la competencia social alude a un juicio valorativo referido a la calidad del comportamiento social del sujeto en un determinado contexto.

Otro término muy empleado es el de asertividad, que ha sido utilizado para referir a conductas de autoafirmación y expresión de sentimientos. Se considera necesario analizarlo a fin de determinar el grado de superposición que puede tener con el concepto de HHSS. Se entiende por asertividad la posibilidad que tiene un sujeto de expresar adecuadamente las emociones en las relaciones sociales, es decir que al manifestarse deben estar ausentes signos de agresividad o ansiedad (Da Dalt de Mangione \& Difabio de Anglat, 2002; Estrada, 2010). Es Wolpe (1977) quien inaugura el término asertividad, y posteriormente se fueron haciendo reelaboraciones del concepto y consideraciones sobre los alcances del mismo. Hace referencia a la capacidad de autoafirmación en el contexto de las relaciones interpersonales. Caballo (2000) puntualiza que la asertividad es la capacidad de un sujeto para expresar pensamientos o sentimientos a otros de un modo directo, efectivo y apropiado. A su vez, el constructo asertividad se inserta en otro denominado prosocialidad. Pithod (1993) señala que los sujetos con motivación altruista, denominada también prosocial genuina, son proclives a desarrollar una personalidad sana y asertiva (Mc Loughlin, 2009).

Finalmente, cabe señalar que existe coincidencia en diversos autores (Paula, 2000; Caballo, 1993; Da Dalt de Mangione \& Difabio de Anglat, 2002; Monjas, 2000) acerca de que el concepto asertividad es restringido y que se integra dentro de otro más amplio, el de habilidades sociales. Numerosas investigaciones han puesto de manifiesto la sólida relación existente entre un adecuado funcionamiento social y el éxito en la vida cotidiana (Hops \& Greenwood, 1998; Ladd \& Asher, 1985; Sánchez, Cerezo, 2010). La adolescencia es un periodo del ciclo vital en el que las habilidades sociales son un factor fundamental para el ajuste social. Es Bandura quien realiza aportes valiosos acerca de la importancia de las variables sociales en el aprendizaje en la niñez y en la adolescencia (Bandura \& Walters, 1978; Bandura, 2002). Aplicado este concepto al campo de las HHSS, se dirá que las mismas se aprenden en un contexto y que una determinada habilidad puede ser valorada en un grupo cultural y no en otro. 
Del Bosque \& Aragón (2008) plantean que el lograr un ajuste entre características personales -incluyendo sus necesidades- y las demandas del medio donde interactúa el adolescente, debe ser entendido en función de los cambios que se atraviesan. En medio de estos cambios, los adolescentes tienen que lograr desarrollar conductas que les permitan adaptarse a los diferentes ambientes en los que se desenvuelven y sentirse satisfechos al lograr también ajustar su propio comportamiento a lo que ellos mismos necesitan. Así, un adecuado autoconcepto y autoestima, facilitarían tanto la interacción familiar como el logro académico. En esta línea de pensamiento, Llinares, Molpeceres \& Mucito (2001) afirman que los adolescentes de alta autoestima académica tienden a priorizar los valores prosociales. Según Caballo (2007) los sujetos más hábiles socialmente presentarían mayor frecuencia, cantidad y duración en contacto visual, sonrisa y expresión facial general, postura, volumen de voz, duración del habla. Los menos sociables realizan menor contacto visual, sonrisas, gestos, mayor índices de ansiedad y presencia de conductas de consentimiento. Camacho \& Camacho (2005) coinciden con Caballo (2007) al considerar que no existe criterio absoluto de habilidad social. El sujeto socialmente hábil es seguro de sí mismo, capaz de autoafirmarse y de responder correctamente a los demás. Garaigordobil \& García de Galdeano (2006), en un estudio con sujetos de 10 a 12 años sobre la empatía -entendida como la respuesta a los demás teniendo en cuenta aspectos cognitivos y afectivos, pudiendo discriminar lo propio y lo ajeno-, hallaron que los sujetos con alta empatía presentan más conductas sociales positivas como consideración, autocontrol y liderazgo; con pocas conductas sociales negativas como agresividad, impulsividad, antisocial y retraimiento.

Dentro de las habilidades sociales se reconocen diferentes estilos. El asertivo permite el establecimiento de relaciones sociales significativas y una mejor adaptación social. En cambio otros estilos de vinculación, tales como el agresivo y el inhibido, dan cuenta de déficit en las habilidades sociales. (Caballo, 1993; 1987; Godoy, Gavino, Blanco, Martorell, Navarro \& Silva 1993; González, Ramos, Caballero \& Wagner, 2003; Matson, Rotatori \& Helsel, 1983). Tantos los adolescentes inhibidos como los impulsivos presentan dificultades en las interacciones sociales (Garaigordobil, 2004; Torrente \& Rodríguez, 2004).

\section{Habilidades sociales y contexto de procedencia}

En cuanto al contexto de procedencia, si bien no se registra suficiente bibliografía con referencias a las relaciones entre nivel socioeconómico alto y habilidades sociales, existe con- 
senso entre los investigadores en la idea que diferentes contextos sociales se asocian a distintos resultados en la competencia social en la infancia y la adolescencia (Soriano \& Soriano, 1994). El contexto impacta sobre los factores de riesgo de dos maneras: o bien aportando factores protectores, a través de un sistema de apoyo (normas y valores) que ayudan al niño o al adolescente en la autoafirmación del yo; o debilitando al sujeto cuando falla en la protección de los factores sociales y culturales hostiles, o por procesos de exclusión de la sociedad dominante (Roberts, Garritz \& Kearey, 1990).

En un estudio sobre la influencia de variables sociodemográfica en población adolescente de centros públicos y privados españoles Paz, Teva \& Buela-Casal (2009) hallaron diferencias significativas a favor de los primeros en relación a estrategias de afrontamiento de solución de problemas y de relación con los demás. En esta línea, Trianes, Cardelle-Elawar, Blanca \& Muñoz (2003) parten de la idea que el contexto social produce más diferencias que el género en relación a las habilidades sociales. En sus investigaciones encontraron que adolescentes de 11 y 12 años de zonas residenciales, según términos de los autores, de Andalucía puntúan más alto en agresividad hacia los iguales, que los adolescentes que viven en contextos deprimidos. Sin embargo, los mismos investigadores encontraron que los alumnos de niveles socioeconómicos altos evidencian puntuaciones más elevadas en asertividad y más bajo en agresividad y pasividad en las relaciones con los adultos. Por otra parte, estudios realizados en Brasil, mostraron una acentuada prevalencia de dificultades en la competencia social en adolescentes pertenecientes a grupos familiares monoparentales de nivel socioeconómico bajo (Assis, Avanci \& Oliverira, 2009). En Argentina, un 12\% de niños de 6 a 11 años registraron algún tipo de problemas sociales y el $16 \%$ con problemas agresivos. En este mismo estudio los resultados epidemiológicos efectuados por el Ministerio de Salud de la Nación conjuntamente con las Universidades Nacionales, mostraron que en los niños de NES bajo se encontraron las mayores frecuencias en cuanto a la presencia de comportamientos antisociales y agresividad y a su vez, en las provincias de mayor frecuencia de éstos problemas fueron $\mathrm{Tu}-$ cumán, Córdoba y San Luis (Ministerio de Salud de la Nación, 2007).

\section{Habilidades sociales y género}

En el desarrollo social, las distintas culturas imprimen patrones de comportamiento que se consideran distintivos y propios de hombres y mujeres; esto es roles de género. Los roles de género son un conjunto de comportamientos y creencias que conforman según Mon- 
jas (2004) los estereotipos de roles de género acerca de lo que socialmente se espera apropiado para hombres y mujeres (Muñoz, Jiménez \& Moreno, 2008). Cabe destacar que las investigaciones sobre diferencias de género y habilidades sociales en la infancia y adolescencia son más bien escasas. Caballo (2007) sostiene que la literatura sobre habilidades sociales y diferencias de género es inconsistente. Los resultados no apoyan la hipótesis de que el comportamiento social esté influido por los roles de género, sino por el contrario, pareciera estar condicionado por la respuesta de los demás ante un sujeto socialmente habilidoso o no habilidoso.

\section{Objetivos}

Los objetivos del presente estudio fueron: a) describir las habilidades sociales en adolescentes de 11 y 12 años de nivel socioeconómico bajo y analizar la prevalencia de las escalas facilitadotas o inhibidoras de la socialización, b) describir las habilidades sociales en adolescentes de 11 y 12 años de nivel socioeconómico alto y analizar la prevalencia de las escalas facilitadotas o inhibidoras de la socialización, c) Comparar la cualidad de las HHSS de los adolescentes de NES bajo con respecto a las HHSS de los adolescentes de NES alto, d) Comparar las HHSS de los adolescentes según sexo y NES (alto/bajo).

\section{Método}

\section{Participantes}

Un total de 283 adolescentes de 11 y 12 años de nivel socioeconómico (NES) bajo y alto (153 adolescentes de NES Bajo y 130 adolescentes de NES Alto), asistentes a $6^{\circ}$ año de educación general básica, EGB 3 (turno mañana) de cuatro escuelas del ámbito público y privado, de San Miguel de Tucumán (Tucumán, Argentina) ${ }^{1}$.

\section{Instrumentos}

Las variables objeto de estudio son habilidades sociales, nivel socioeconómico y género. La evaluación de las habilidades sociales, se realizó a partir de la Escala BAS - 3: Batería

\footnotetext{
${ }^{1}$ Proyecto $26 \mathrm{~K} 403$ Habilidades sociales y habilidades cognitivas en adolescentes que viven bajo condiciones de pobreza. Un estudio comparativo. Directora Dra. E. Norma Contini. Financiado por Consejo de Investigaciones Universidad Nacional de Tucumán, (CIUNT).
} 
de Socialización de Silva y Martorell (1994). Es un cuestionario Autoadministrable para adolescentes de 11 a 19 años. Evalúa 5 dimensiones de la conducta social: Consideración con los demás (Co); Autocontrol (Ac); Retraimiento Social (Re). Ansiedad Social / Timidez (At); Liderazgo (Li). Cuenta con una escala de Sinceridad (S), que fue incluida con fines vinculados a la confiabilidad de la Batería. Un sujeto socialmente hábil corresponde a aquel que obtiene percentil mayor o igual a 75 en escalas Consideración con los demás (Co), Autocontrol en las relaciones sociales (Ac) y Liderazgo (Li); y sus percentiles son iguales o menores a 25 en las escalas Retraimiento social (Re) y Ansiedad social/Timidez (At).

Con respecto a la estructura de la prueba, se realizó un análisis factorial con una muestra de población española. A partir de 92 elementos se detectó una estructura clara de cinco factores, pero pertenecen al cuestionario los sesenta y cinco ítems de más alta saturación, a los que se agregaron diez para valorar Sinceridad. Algunas de las escalas poseen una distribución irregular, por lo que se realizó una baremización de distribución libre. Las escalas presentaron relativa independencia entre sí, aunque se registró un patrón de intercorrelaciones entre las mismas que permite una clara interpretación desde sus contenidos. Las correlaciones altas y negativas se dieron entre Consideración con los demás (Co) y Retraimiento $(\mathrm{Re})$, lo que es coherente ya que la consideración hacia los demás es opuesta con la inhibición social o el retraimiento. Co correlaciona positivamente con Autocontrol (Ac) y Liderazgo (Li); esto es, la sensibilidad social se vincula con acatamiento a reglas de convivencia social y con espíritu de servicio. Se encontró una correlación entre Ansiedad (At) y Retraimiento Social (Re); entre At y Li fue baja y negativa, a menor nerviosismo y timidez por la relación social mayor confianza en sí mismo, popularidad, y viceversa. En general, las escalas facilitadoras de la socialización correlacionan positivamente con Sinceridad.

Las puntuaciones directas se transforman en percentiles. La interpretación se realiza en sentido contrario según se trate de escalas facilitadoras de la socialización (Co, Ac y Li) o perturbadoras de la misma (Re y At). Es decir, en las facilitadoras los percentiles superiores al término medio ( $P 75$ o más) muestran mayor nivel de habilidades sociales. En las inhibidoras este rango percentilar está indicando déficit en las habilidades sociales. La confiabilidad de la BAS-3 se encuentra dentro de límites satisfactorios teniendo en cuenta el número de ítem (los valores de las 5 escalas varían de .60 a .82). La variabilidad temporal es irregular, por lo que los resultados en estudios longitudinales deben considerarse con reservas. 
La encuesta sociodemográfica fue administrada para identificar el nivel socioeconómico en los grupos objetos de estudio. Se tomaron como indicadores el nivel de ocupación y educación del jefe del hogar, hacinamiento, estructura del grupo familiar con el que convivía el examinado, lugar de residencia y posesión de determinados bienes. El NES - alto, bajo -se determinó por la combinación de ocupación, educación y acceso a bienes de consumo (Cámara de Empresas de Investigación Social y de Mercado y Asociación Argentina de Marketing, 2006). La variable género fue evaluada como un ítem de la encuesta, a la que el alumno responde indicando si es varón o mujer.

\section{Procedimiento}

Se aplicó la Batería de Socialización BAS- 3 y la Encuesta Sociodemográfica a los alumnos de $6^{\circ}$ año de EGB 3, turno mañana. En función de las características de la población y la accesibilidad, se seleccionaron cuatro escuelas de NES Bajo y Alto. Tarea realizada por el equipo de investigación y alumnos avanzados de la carrera de Psicología de la Universidad Nacional de Tucumán (UNT), entrenados a tal fin. Las puntuaciones obtenidas fueron analizadas con el PASW Statistics 18.

\section{Análisis estadísticos}

Para el análisis estadístico se realizaron pruebas específicas de la función descriptiva para variables cuantitativas, tales como Medias Aritméticas y Desviaciones típicas de cada una de las escalas de la BAS-3, considerando dos grupos independientes; uno para el NES alto y otro para el NES bajo. También se especificaron los valores máximos y mínimos de cada escala.

Se realizaron también pruebas bivariadas de correlación (coeficiente $\mathrm{r}$ de Pearson) y estadística inferencial para evaluar el nivel de significación en las diferencias de las medias de ambos NES, lo cual implicó la estimación de igualdad entre las muestras (Prueba de Levene) y el cálculo de los límites del intervalo de confianza del 95\%.

\section{Resultados}




\section{La socialización en adolescentes}

El primer objetivo, fue describir las habilidades sociales en los adolescentes participantes de NES bajo. Para ello se realizó un análisis descriptivo de las escalas facilitadoras de la socialización, encontrándose un mayor desempeño en la escala Consideración con los demás, Co $(M=11.42$; DT= 2.29), y en la de Liderazgo, Li $(M=8.04 ; \mathrm{DT}=2.07)$. Con respecto a las escalas inhibidoras de la socialización se encontró un mayor desempeño en la Escala de Ansiedad/Timidez, At (M=9.22; DT=2.59). Ver Tabla 1.

Tabla 1. Descripción de Escalas BAS-3 según estadísticos descriptivos. Adolescentes NES

\begin{tabular}{lrrrrrr}
\multicolumn{5}{c}{ Bajo. Provincia de Tucumán, Argentina } \\
\hline \multicolumn{1}{c}{ ESCALAS } & BAS-3 & Media & $\begin{array}{c}\text { Desv. } \\
\text { Estándar }\end{array}$ & Mín & Máx \\
\hline $\begin{array}{l}\text { Consideración } \\
\text { demás }\end{array}$ & con los & 11.42 & 2.29 & 4 & 14 \\
$\begin{array}{l}\text { Autocontrol en las } \\
\text { ciones sociales }\end{array}$ & & 9.22 & 2.59 & 1 & 14 \\
$\begin{array}{l}\text { Retraimiento Social } \\
\text { Ansiedad Social / Timidez }\end{array}$ & 4.00 & 2.14 & 0 & 12 \\
Liderazgo & 5.71 & 2.63 & 0 & 12 \\
\hline
\end{tabular}

El segundo objetivo fue, describir las habilidades sociales en adolescentes de 11 y 12 años de NES Alto. Se realizó un análisis descriptivo de las escalas facilitadoras de la socialización, a partir del cual se encontró un mayor desempeño de los adolescentes en la escala Consideración con los demás, Co $(\mathrm{M}=12.35$; DT= 2.42) y en la escala de Autocontrol, Ac (M 9.94; DE 3.03) que en la escala de Liderazgo, $\mathrm{Li}(\mathrm{M}=6.98 ; \mathrm{DT}=2.63)$. Con respecto a las escalas denominadas inhibidoras de la socialización, el desempeño fue mejor en la escala Retraimiento, $\operatorname{Re}(\mathrm{M}=1.52 ; \mathrm{DT}=2.06)$ que en la escala Ansiedad / Timidez, At $(\mathrm{M}=3.95 ; \mathrm{DT}$ 2.86). Ver Tabla 2. 
Tabla 2. Descripción de Escalas BAS-3 según estadísticos descriptivos. Adolescentes NES

\begin{tabular}{lrrrrrr}
\multicolumn{5}{c}{ Alto. Provincia de Tucumán, Argentina } \\
\hline \multicolumn{1}{c}{ ESCALAS } & BAS-3 & Media & $\begin{array}{c}\text { Desv. } \\
\text { Estándar }\end{array}$ & Mín & Máx \\
\hline $\begin{array}{l}\text { Consideración con los } \\
\text { demás }\end{array}$ & 12.35 & 2.42 & 0 & 14 \\
$\begin{array}{l}\text { Autocontrol en las } \\
\text { ciones sociales }\end{array}$ & 9.94 & 3.03 & 0 & 14 \\
$\begin{array}{l}\text { Retraimiento Social } \\
\text { Ansiedad Social / Timidez }\end{array}$ & 1.52 & 2.06 & 0 & 12 \\
Liderazgo & 6.98 & 2.86 & 0 & 12 \\
\hline
\end{tabular}

Posteriormente se realizaron correlaciones bivariadas entre las distintas escalas de la BAS-3. Se encontró una asociación estadísticamente significativa entre Consideración con los demás (Co) y Autocontrol (Ac) (r 0.357, $p<.0001)$. Ver Tabla 3.

Tabla 3. Análisis bivariado entre las Escalas del BAS-3. Adolescentes NES Alto y Bajo. Provincia de Tucumán, Argentina.

\begin{tabular}{|c|c|c|c|c|c|c|}
\hline & $\begin{array}{l}\text { Considera- } \\
\text { ción con los } \\
\text { demás }\end{array}$ & $\begin{array}{l}\text { Auto- } \\
\text { control }\end{array}$ & $\begin{array}{l}\text { Retraimien- } \\
\text { to }\end{array}$ & $\begin{array}{c}\text { Ansie- } \\
\text { dad/Timidez }\end{array}$ & $\begin{array}{l}\text { Lideraz- } \\
\text { go }\end{array}$ & $\begin{array}{l}\text { Sinceri- } \\
\text { dad }\end{array}$ \\
\hline \multirow{2}{*}{$\begin{array}{l}\text { Considera- } \\
\text { ción con los } \\
\text { demás }\end{array}$} & 1 & ,412** &,$- 327 * *$ &,- 052 & ,349** &,- 080 \\
\hline & & ,000 & ,000 &, 364 &, 000 & ,161 \\
\hline \multirow{2}{*}{ Autocontrol } &, $412 * *$ & 1 &,$- 263 * *$ &,$- 176^{* *}$ &,- 063 &,- 061 \\
\hline & ,000 & & ,000 &, 002 & ,267 & ,282 \\
\hline \multirow[t]{2}{*}{ Retraimiento } &,$- 327 * *$ &,$- 263 * *$ & 1 &, $418 * *$ &,- 063 &,- 061 \\
\hline &, 000 & ,000 & & ,000 & ,267 & ,282 \\
\hline \multirow{2}{*}{$\begin{array}{c}\text { Ansie- } \\
\text { dad/Timidez }\end{array}$} &,- 052 &,$- 176 * *$ &, $418 * *$ & 1 &,- 072 & 034 \\
\hline & ,364 &, 002 & ,000 & & ,202 &, 546 \\
\hline \multirow{2}{*}{ Liderazgo } &, $349 * *$ &, $171 * *$ &,- 063 &,- 072 & 1 &,$- 205^{* *}$ \\
\hline & ,000 & ,002 & ,267 & ,202 & &, 000 \\
\hline \multirow{2}{*}{ Sinceridad } &,- 080 &,$- 354 * *$ &,- 061 & 034 &,$- 205^{* *}$ & 1 \\
\hline & ,161 & ,000 & ,282 &, 546 &, 000 & \\
\hline
\end{tabular}

** La correlación es significativa al nivel 0,001 (bilateral) 
En las escalas inhibidoras de la socialización Re y At, las puntuaciones más elevadas se encontraron en los adolescentes de NES Bajo, lo que estaría indicando mayores índices de apartamiento de los demás $(\mathrm{t}=9.699 ; \alpha<0.001)$.

El tercer objetivo de este estudio fue comparar la cualidad de las HHSS de los adolescentes de NES bajo participantes con respecto a las HHSS de sus pares de NES alto. Los análisis univariados (Prueba T para muestras independientes) mostraron diferencias estadísticas significativas en las escalas de Consideración con los demás, Co, $(\mathrm{t}(\mathrm{gl}=281)=3.084, \mathrm{p}=$ .002), Liderazgo, Li, $(\mathrm{t}(\mathrm{gl}=281)=-4.364, \mathrm{p}=.000)$, Retraimiento, $\mathrm{Re},(\mathrm{t}(\mathrm{gl}=281)=-9.699, \mathrm{p}$ $=.000)$ y Ansiedad/timidez, At, $(\mathrm{t}(\mathrm{gl}=281)=-5.715, \mathrm{p}=.000)$. Estas diferencias estadísticas también se registraron en la escala Sinceridad, $\mathrm{S},(\mathrm{t}(\mathrm{gl}=281)=5.871, \mathrm{p}=.000)$ Ver Tabla 4.

Tabla 4. Diferencia de Medias (t) para las Escalas del BAS-3 entre los adolescentes de NES Alto y Bajo. Provincia de Tucumán, Argentina.

\begin{tabular}{|c|c|c|c|c|c|c|c|c|c|c|}
\hline & & \multicolumn{4}{|c|}{$\begin{array}{c}\text { Prueba de Levene } \\
\text { para la igualdad } \\
\text { de varianzas }\end{array}$} & \multicolumn{3}{|c|}{ Prueba T para la igualdad de medias } & & \\
\hline & & \multirow[b]{2}{*}{$F$} & \multirow[b]{2}{*}{ Sig. } & \multirow[b]{2}{*}{$\mathrm{t}$} & \multirow[b]{2}{*}{$\mathrm{gl}$} & \multirow{2}{*}{$\begin{array}{c}\text { Sig. } \\
\text { (bilateral) }\end{array}$} & \multirow{2}{*}{$\begin{array}{l}\text { Diferencia } \\
\text { de medias }\end{array}$} & \multirow{2}{*}{$\begin{array}{l}\text { Error típ. de } \\
\text { la diferencia }\end{array}$} & \multicolumn{2}{|c|}{$\begin{array}{c}95 \% \text { Intervalo de } \\
\text { confianza para la } \\
\text { diferencia } \\
\end{array}$} \\
\hline & & & & & & & & & Inferior & Superior \\
\hline CONSIDERACION & Se han asumido & 3,332 & 069 & 3,084 & 281 & 002 & ,883 & ,286 & ,320 & 1,447 \\
\hline \multirow[t]{2}{*}{ CON LOS DEMÁS } & varianzas iguales & & & & & & & & & \\
\hline & $\begin{array}{l}\text { No se han asumido } \\
\text { varianzas iguales }\end{array}$ & & & 3,080 & 272,078 & ,002 & ,883 & ,287 & ,319 & 1,448 \\
\hline \multirow[t]{2}{*}{ AUTOCONTROL } & $\begin{array}{l}\text { Se han asumido } \\
\text { varianzas iguales }\end{array}$ & 984 & ,322 & 1,701 & 281 & ,090 &, 566 & ,333 &,- 089 & 1,221 \\
\hline & $\begin{array}{l}\text { No se han asumido } \\
\text { varianzas iguales }\end{array}$ & & & 1,678 & 253,388 &, 095 &, 566 &, 337 &,- 098 & 1,230 \\
\hline \multirow[t]{2}{*}{ LIDERAZGO } & $\begin{array}{l}\text { Se han asumido } \\
\text { varianzas iguales }\end{array}$ & 5,039 & ,026 & $-4,364$ & 281 & ,000 & $-1,232$ & ,282 & $-1,788$ &,- 676 \\
\hline & $\begin{array}{l}\text { No se han asumido } \\
\text { varianzas iguales }\end{array}$ & & & $-4,288$ & 246,254 &, 000 & $-1,232$ & ,287 & $-1,798$ & -666 \\
\hline \multirow[t]{2}{*}{ RETRAIMIENTO } & $\begin{array}{l}\text { Se han asumido } \\
\text { varianzas iguales }\end{array}$ & 2,403 & , 122 & $-9,699$ & 281 & ,000 & $-2,472$ & ,255 & $-2,973$ & $-1,970$ \\
\hline & $\begin{array}{l}\text { No se han asumido } \\
\text { varianzas iguales }\end{array}$ & & & $-9,749$ & 278,218 & ,000 & $-2,472$ & ,254 & $-2,971$ & $-1,973$ \\
\hline $\begin{array}{l}\text { ANSIEDAD / TIMI- } \\
\text { DEZ }\end{array}$ & $\begin{array}{l}\text { Se han asumido } \\
\text { varianzas iguales }\end{array}$ & ,580 & ,447 & $-5,715$ & 281 & ,000 & $-1,877$ & ,328 & $-2,524$ & $-1,231$ \\
\hline
\end{tabular}




\begin{tabular}{|c|c|c|c|c|c|c|c|c|c|c|}
\hline & $\begin{array}{l}\text { No se han asumido } \\
\text { varianzas iguales }\end{array}$ & & & $-5,680$ & 265,659 & ,000 & $-1,877$ & ,331 & $-2,528$ & $-1,227$ \\
\hline \multirow[t]{2}{*}{ SINCERIDAD } & $\begin{array}{l}\text { Se han asumido } \\
\text { varianzas iguales }\end{array}$ & 1,030 &, 311 & 5,871 & 281 &, 000 & 1,566 & ,267 & 1,041 & 2,092 \\
\hline & $\begin{array}{l}\text { No se han asumido } \\
\text { varianzas iguales }\end{array}$ & & & 5,842 & 267,515 &, 000 & 1,566 & ,268 & 1,038 & 2,094 \\
\hline
\end{tabular}

Habilidades Sociales y género

Posteriormente se llevó a cabo un análisis multivariado de la varianza (MANOVA) con un diseño 2 ( ticamente significativas entre la medias de los grupos respecto al sexo (Lambda de Wilks = $.933, F(5,275)=3.96, p=.002)$ y al NES (bajo/alto) $($ Lambda de Wilks $=.622, F(5,275)=$ 33.488, $p=.001$ ). Asimismo se observó interacción entre sexo y NES (Lambda de Wilks = $.024, F(5,275)=2271.55=.000$.

Los análisis univariados respecto a la combinación sexo y NES indicaron que las mujeres del NES bajo presentaron mayores índices de Ansiedad/Timidez, At que sus pares del sexo opuesto. Mientras que los varones del NES alto mostraron mayor Retraimiento, Re. Ver Tabla 5 .

Tabla 5. Medias, Desviaciones Típicas y Pruebas de diferencias de medias entre adolescentes de diferente sexo y Nivel Económico y Social en las Escalas BAS-3.

\begin{tabular}{cccccc}
\hline Escalas & \multicolumn{2}{c}{ Mujeres } & \multicolumn{2}{c}{ Varones } & $\mathrm{F}$ \\
BAS-3 & NES bajo & NES alto & NES bajo & NES alto & \\
& $\mathrm{n}=62)$ & $\mathrm{N}=68$ & $\mathrm{n}=62$ & $\mathrm{n}=62$ & \\
$\mathrm{Co}$ & 11.31 & 12.63 & 11.08 & 12.05 & $742.16 * * *$ \\
& $(2.60)$ & $(2.30)$ & $(2.31)$ & $(2.53)$ & \\
$\mathrm{Ac}$ & 9.45 & 10.13 & 8.81 & 9.73 & $389 * * *$ \\
& $(2.67)$ & $(3.16)$ & $(2.42)$ & $(2.91)$ & \\
$\mathrm{Re}$ & 4,05 & 1.16 & 4.50 & 1.90 & $26.51 * * *$ \\
& $(2.21)$ & $(1.64)$ & $(2.22)$ & $(2.39)$ & \\
$\mathrm{At}$ & 6.44 & 4.29 & 5.31 & 3.56 & $154.17 * * *$ \\
& $(2.60)$ & $(3.01)$ & $(2.74)$ & $(2.68)$ & \\
$\mathrm{Li}$ & 1.77 & 6.99 & 2.26 & 6.97 & $279.14 * * *$ \\
& $(2.88)$ & $(2.66)$ & $(2.37)$ & $(2.63)$ & \\
\hline
\end{tabular}

Nota: $* * * \mathrm{p}<.001$ 


\section{Discusión y conclusiones}

El primer objetivo fue describir las habilidades sociales en los adolescentes participantes de NES bajo. Los resultados encontrados demuestran que presentan sensibilidad social y preocupación por los demás, en particular por quienes se encuentran frente a situaciones problemáticas. Por su significación se ha denominado a esta dimensión (Co) facilitadora de la socialización. Prosiguiendo con el análisis de otra dimensión de la conducta social, en la línea de las llamadas facilitadoras, en Liderazgo ( $\mathrm{Li}$ ) el adolescente tiene un rol protagónico en el grupo en el cual se desenvuelve, ascendencia entre sus pares, iniciativa y capacidad para organizar y conducir actividades con sus iguales.

En cuanto al segundo de los objetivos estudiados, se encontró una asociación estadísticamente significativa entre Consideración con los demás $(\mathrm{Co})$ y Autocontrol $(\mathrm{Au})$. Estos resultados son semejantes a los obtenidos por los autores de la prueba (Silva \& Martorell, 2001) y son consistentes con la significación de las escalas. Por cuanto Autocontrol (Ac), en su polo positivo implica aceptación de normas sociales y respeto por el otro, es coherente la presencia de puntuaciones en el mismo sentido en Consideración con los demás (Co), que informa si el adolescente actúa con sensibilidad social e interés por el prójimo. A su vez, estos resultados dan cuenta que estos adolescentes tienden a priorizar valores prosociales, en donde la comunicación y la cooperación entre pares promueven la sociabilidad (Garaigordobil, 2001; Trianes, 1996).

En cuanto al tercer objetivo, comparar la cualidad de las HHSS de los adolescentes según contexto, en el grupo de adolescentes de NES Bajo, las puntuaciones más elevadas se encontraron en la Escala de Retraimiento (Re) y en la de Ansiedad/Timidez (At). Estas dimensiones corresponden a las escalas inhibidoras de la socialización. Como se indicó en la sección Método, teniendo en cuenta los percentiles 25 y 75, su interpretación se realiza en sentido contrario según se trate de escalas facilitadoras de la socialización (Co, Ac, y Li) o inhibidoras de la misma (Re y At).

Estos resultados dan cuenta que los adolescentes de NES Bajo, en comparación con los adolescentes de NES Alto, presentan manifestaciones de temor en las relaciones sociales, unidas a timidez y sentimientos de aislamiento social. Estos comportamientos son disfuncionales, pueden afectar la salud integral de estos adolescentes y tal como señala Trianes (1996) 
llegar a constituirse en factores de riesgo. Como se sabe el trastorno de fobia social y los trastornos de ansiedad suelen estar mal diagnosticados o no ser reconocidos (Estrada, 2010) por los que los resultados encontrados cobran especial relevancia a los fines de la atención en salud mental del adolescente. A su vez, queda abierto el interrogante acerca de qué manera las condiciones de vida (bajo nivel de escolaridad del jefe del hogar, empleo no calificado del mismo, habitat inadecuado, escaso acceso a los bienes de la cultura) impactan en el adolescente y generan esta modalidad de vinculación y cuáles son los factores protectores que operan positivamente en quienes no han desarrollado este comportamiento.

También se encontró que éstos, en relación a sus pares de NES Alto, puntuaron significativamente más alto en la escala facilitadora de Liderazgo (Li). La incongruencia de las relaciones obtenidas entre Liderazgo (Li) y Retraimiento $(\mathrm{Re})$ deben ser investigados en estudios posteriores por la presencia de indicadores de baja Sinceridad (S), es decir, el nivel de honestidad con que el adolescente respondió a los reactivos de la prueba. Otra de las limitaciones de la presente investigación es la posible deseabilidad social presente en las respuestas de los adolescentes. Para superar las limitaciones señalas sería necesario el análisis de muestras más amplias.

Diferentes investigaciones han demostrado que las condiciones de pobreza impactan en el capital simbólico y cultural de las familias en las que han crecido estos adolescentes de NES bajo (Contini, 2008; Da Dalt de Mangione \& Difabio de Anglat, 2002) pero son insuficientes para explicar las habilidades de Liderazgo en este grupo, lo cual requiere una ampliación de estudios en esta línea a fin de contar con mayor información que permita explicar la configuración de esta habilidad en el contexto de pobreza.

En cuanto a los adolescentes de NES Alto, los resultados encontrados dan cuenta de que puntúan más elevado que sus pares de NES Bajo en la escala Consideración con los demás (Co). Demuestran sensibilidad social y preocupación por los demás, en particular por lo que se encuentran frente a situaciones problemáticas. Por su significación se ha denominado a esta dimensión facilitadora de la socialización ya que implica la presencia de comportamientos asertivos. Es decir, la capacidad de expresar pensamientos y sentimientos a otros de una manera efectiva y apropiada, una mayor tendencia a expresarse de forma directa, pero no coercitiva, en situaciones tales como manifestar sentimientos negativos y positivos (Caballo, 1987, 1993, 2000). A su vez la asertividad se inserta en la denominada prosocialidad (Pithod, 
1993) que hace referencia a una motivación altruista. Los sujetos prosociales son proclives a desarrollar una personalidad saludable. Se orienta, entonces, hacia una perspectiva salugénica en la organización psicológica del adolescente. Con respecto a la dimensión Consideración con los demás (Co) se interpreta que los adolescentes con los que se investigó demuestran sensibilidad social y preocupación por los demás, en particular por quienes se encuentran frente a situaciones problemáticas.

Los adolescentes de NES alto, en comparación con sus pares de NES bajo, presentaron comportamientos prosociales, tales como consideración con los demás, preocupación por los que tienen problemas o son rechazados, comportamientos de autocontrol, manifestado por acatamientos de reglas y normas sociales que facilitan la convivencia. Los resultados hallados indicaron que las mujeres del NES bajo presentaron mayores manifestaciones de ansiedad y timidez en las relaciones sociales (Escala de Ansiedad/timidez, At) que sus pares del sexo opuesto. Es decir que las mujeres de este grupo presentaron síntomas de ansiedad social, que se manifiesta en miedo, nerviosismo, timidez e inhibición en las relaciones sociales. Estos resultados difieren de los obtenidos por Monjas (2004), quien no encontró diferencias significativas entre varones y mujeres en las variables Inhibición y Ansiedad. Distintos resultados se encontraron en estudios de Muñoz, Jiménez \& Moreno (2008) quienes, desde una perspectiva de multiobservadores (pares, docentes y el propio sujeto) sobre las relaciones sociales en adolescentes de Sevilla, determinaron que las mujeres obtenían medias más altas en sociabilidad y los varones en agresión e inmadurez, no presentando diferencias en relación a la dimensión aislamiento. En cambio, los varones de NES alto mostraron mayor índice de apartamiento de los demás (Escala de Retraimiento, Re). Éstos evidenciaron pasividad, comportamientos evasivos y de aislamiento social. Estos resultados concuerdan con los hallados por Díaz (2005) quien encontró que existe una relación entre la competencia social y los aspectos relacionales en el aula. Es así, que los profesores evalúan a los niños menos habilidosos socialmente que las niñas.

Finalmente, se destaca que estos resultados aportan información útil a la hora de diseñar estrategias de intervención que operen tanto en los déficits de los comportamientos sociales, como así también, en el fortalecimiento de comportamientos saludables. Estas acciones, con seguridad, van a contribuir a la prevención de futuros comportamientos psicopatológicos. 


\section{Referencias}

Ávila-Espada, A., Jiménez-Gómez, F. y González, M. (1996). Aproximación psicométrica a los patrones de personalidad y estilos de afrontamiento del estrés en la adolescencia: perspectivas conceptuales y técnicas de evaluación. En M. M. Casullo (Comp.) Evaluación psicológica en el campo de la salud (pp. 267-325). Barcelona: Paidós-Ibérica.

Assis, S., Avanci, J. y Oliveira, R. (2009). Desigualdades socioeconómicas y salud mental infantil. Saúde Publica, 43(1), 92-100.

Bandura, A. y Walters, R. (1978). Aprendizaje social y desarrollo de la personalidad. Madrid: Alianza Editorial.

Bandura, A (2002). Social Cognitive Theory in Cultural Context. Applied Psychology, 51 (2), 269-290.

Caballo, V. (1987). Teoría, evaluación y entrenamiento de las habilidades sociales. Valencia: Promolibro.

Caballo, V. (1993). Relaciones entre diversas medidas conductuales y de autoinforme de las habilidades sociales. Psicología Conductual, 1, 73-99.

Caballo, V. (2000). Manual de evaluación y entrenamiento de habilidades sociales. Madrid: Siglo XXI.

Caballo, V. (2007). Manual de Evaluación y entrenamiento de las habilidades sociales. España, México, Argentina: Siglo XXI.

Camacho, C. y Camacho, M. (2005). Habilidades sociales en la Infancia. Revista Profesional Española de Terapia Cognitivo-Conductual.3; 1-27.

Cámara de Empresas de Investigación Social y de Mercado y Asociación Argentina de Marketing. (2006). Recuperado el 15 de noviembre de 2010. Disponible en http://www.aam.com/publicaciones/inse.2006.asp.

Contini, N. (2008). Las habilidades sociales en la adolescencia temprana. Perspectivas desde la Psicología Positiva, Psicodebate. Psicología, cultura y Sociedad, 9, 45-63.

Da Dalt de Mangione, E. y Di Fabio de Anglat, H. (2002). Asertividad. Su relación con los estilos educativos familiares, Interdisciplinaria, 19(2), 119-140.

Diaz-Aguado, M. (2005). La violencia entre iguales en la adolescencia y su prevención desde la escuela. Psicothema,17(4), 549-558. 
Del Bosque, A.,y Aragón, L. (2008). Nivel de Adaptación en adolescentes Mexicanos. Interamerican Journal of Psychology, 42, 287-297.

Estrada, B. (coord) (2010). Avances en investigación y tratamiento sobre fobia social, depresión y competencia social. Barcelona: Libro Electrónico. Psiquiatría.com.

Fernández-Ballesteros, R. (1994). Evaluación conductual hoy: un enfoque para el cambio en psicología clínica y de la salud. Madrid: Pirámide.

Garaigordobil, M. (2008). Conducta antisocial durante la adolescencia: correlatos socioemocionales, predoctores y diferencias de género, Psicología Conductual, 13, 197-215.

Garaigordobil (2004). Intervención psicológica en la conducta agresiva y antisocial en niños, Psicothema, 16 (3), 429-435.

Garaigordobil, M \& García del Galdeano, P. (2006). Empatía en niños de 10 a 12 años. Psicothema, 2, 180-186.

Gil F. y León, J. (1998). Habilidades Sociales. Teoría, investigación e intervención. Madrid: Síntesis.

Griffa, M. y Moreno, J. E. (2005). Claves para una psicología del desarrollo. Adolescencia., Adultez. Vejez. Buenos Aires: Lugar.

Godoy, A., Gavino, A., Blanco, J., Martorell, M., Navarro, A. y Silva, F. (1993). Escalas de Lugar de Control en Situaciones Académicas (ELC-A). En F. Silva \& C. Martorell (Dirs), EPIJ: Evaluación de la personalidad infantil y juvenil (pp.37-79). Madrid: Mepsa.

González, C., Ramos, L., Caballero, M. y Wagner, F. (2003) Correlatos psicosociales de depresión, ideación suicida e intento suicida en adolescentes mexicanos., Psicothema, 15(4), 524-532.

Hops, H. y Greenwood, C. (1998). Social skills deficits. En E. J. Mash y L. G. Terdal (Eds.) Behavioural assessment of childhood disorders. New Cork: Guildford press, 2nd ed., 263314.

Ladd, G. y Asher, S. (1985) Social skill training and children’s peer relations. In L. L’Abate \& M. A. Milan (Eds.). Handbook of Social Skill Training and Research. New York: Wiley, 219-244.

Llinares, L., Molpeceres, M. y Musito, G. (2001). La autoestima y las prioridades personales de valor. Un análisis de sus interrelaciones en la adolescencia. Anales de Psicología, 17, 
189-200.

Matson, J., Rotatori, A. y Helsel, W. (1983). Development of a rating scale to measure social skills in children: the Matson evaluation of social skills with youngsters (MESSY) Behavior Research Terapy, 21 (49), 335-340.

Mc Fall, R. (1982). A review and reformulation of the concept of social skills. Behavioral assessment, 4, 1-33.

Mc Loughlin, C. (2009). Positive Peer Group Interventions: An alternative to Individualized Interventions for Promoting Prosocial Behavior in Potentially Disaffected Youth. Electronic Journal of Research in Educational Psychology. 7, (3), 1131-1156.

Ministerio de Salud de la Nación (2007). Problemática de Salud Mental en la infancia. Proyecto de investigación. Informe final. Recuperado el 20 de diciembre de 2010. Disponible en http: //www.msal.gov.ar/htm/site/pdf/informe_final.pdf

Monjas, M. (2000). Programa de enseñanza de habilidades de interacción social (PEHIS) para niños y niñas en edad escolar. Madrid: CEPE.

Monjas, M. (2004). Ni sumisas ni dominantes. Los estilos de relación interpersonal en la infancia y en la adolescencia. España: Ministerio de Trabajo y asuntos Sociales.

Muñoz, V., Jiménez, M. C \& Moreno, L. (2008). Las tipologías de estatus sociométrico durante la adolescencia: contraste de distintas técnicas y fórmulas para su cálculo. Psicothema, 20, 665-671.

Musitu, G., Buelga, S. Lila, M. y Cava, M. J. (2004). Familia y adolescencia. Madrid: Síntesis.

Paula, I. (2000). Habilidades sociales: educar hacia la autorregulación. Conceptualización, evaluación e intervención. Barcelona: IRCE-Horsori

Paz, T. y Buela-Casal, G. (2009). Influencia de variables sociodemográficas sobre estilos de afrontamiento, el estrés social y la búsqueda de sensaciones sexuales en adolescentes. Psicothema, 21(2), 220-226.

Pelechano, V., Báquena Puigcever, M. y García Pérez, L. (1996). Psicología de la Personalidad. Barcelona: Ariel.

Pithod, A. (1993). Adaptación y estandarización de test para uso en comportamiento organizacional. Mendoza, Argentina. Archivos Centro de Investigaciones Cuyo (CIC). 
Roberts, L., Garritz, B. \& Kearey, K. (1990). Shaping your school's culture to improve student learning. Los Ángeles, CA: California School Leadership Academy.

Sánchez, C., Cerezo, F. (2010). Variables personales y sociales relacionadas con la dinámica bullying en escolares de educación primaria. Electronic Journal of Research in educational Psychology, 8 (3), 1015-1032.

Silva, F. y Martorell, M. (2001). BAS-3 Batería de Socialización. Manual. Madrid: TEA.

Soriano, M. y Soriano, F. (1994). School violence among culturally diverse populations: Sociocultural and institutional considerations. School Psychology Review, 23 (2), 216235.

Trianes, M. (1996). Educación y competencia social: un programa en la escuela. Málaga: Aljibe.

Trianes, M., Cardelle-Elawer, M., Blanca, M. y Muñoz, A. (2003). Contexto Social, género y competencia social autoinformadas en alumnos andaluces de 11 y 12 años. Electronic Journal of Research in Educational Psychology, 1 (2), 37-55.

Torrente, G. y Rodríguez, F. (2004). Características sociales y familiares vinculadas al desarrollo de la conducta delictiva en pre-adolescentes y adolescentes. Cuadernos de trabajo social, 17, 99-115.

Urresti, M. (2008). Ciberculturas juveniles. Buenos Aires: La crujía

Wolpe, J. (1977). Práctica de la terapia de conducta. México: Trillas. 
Coronel, C. P. et al.

[Página en blanco por razones de paginación] 